NASA Technical Memorandum 103958

$$
\text { 1N-OY }
$$

128387

P.II

\title{
Integration of Radar Altimeter, Precision Navigation, and Digital Terrain Data for Low-Altitude Flight
}

Richard E. Zelenka

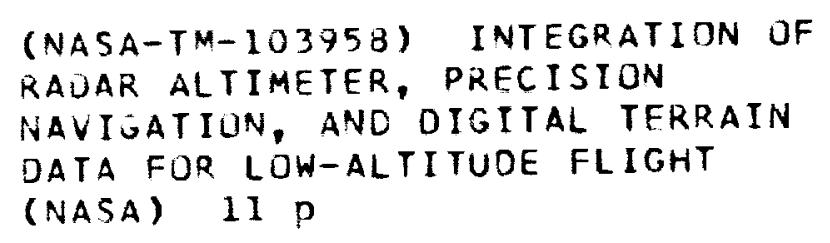

N93-12320

Unclas

DATA FOR LOW-ALTITUDE FLIGHT
(NASA) 1 I

G3/04

0128387

August 1992 


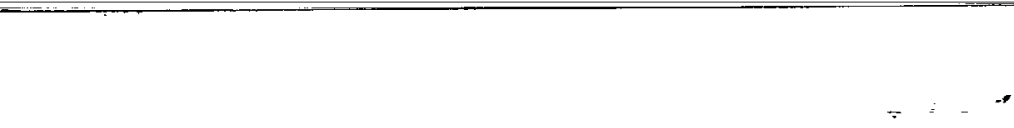

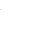

.

$\cdot$

.

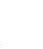

.

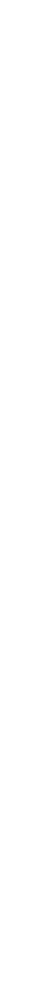




\section{Integration of Radar Altimeter, Precision Navigation, and Digital Terrain Data for Low-Altitude Flight}

Richard E. Zelenka, Ames Research Center, Moffett Field, California

August 1992

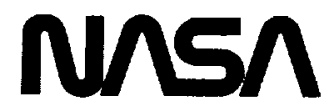

National Aeronautics and

Space Administration 
. . 


\section{Summary}

Avionic systems that depend on digitized terrain elevation data for guidance generation or navigational reference require accurate absolute and relative distance measurements to the terrain, especially as they approach lower altitudes. This is particularly exacting in low-altitude helicopter missions, where aggressive terrain hugging maneuvering create minimal horizontal and vertical clearances and demand precise terrain positioning. Sole reliance on airborne precision navigation and stored terrain elevation data for above-ground-level (AGL) positioning severely limits the operational altitude of such systems. A Kalman filter is presented which blends radar altimeter returns, precision navigation, and stored terrain elevation data for AGL positioning. The filter is evaluated using low-altitude helicopter flight test data acquired over moderately rugged terrain. The proposed Kalman filter is found to remove large disparities in predicted AGL altitude (i.e., from airborne navigation and terrain elevation data) in the presence of measurement anomalies and dropouts. Previous work suggested a minimum clearance altitude of $220 \mathrm{ft}$ AGL for a near-terrain guidance system; integration of a radar altimeter allows for operation of that system below $50 \mathrm{ft}$, subject to obstacle-avoidance limitations.

\section{Introduction}

Numerous avionic systems employ a terrain elevation database for navigation or guidance. Terrain referenced navigation systems, such as SITAN (Sandia Inertial Terrain-Aided Navigation) or TERPROM (TERrain PROfile Matching), utilize radar altimeter returns, a terrain elevation database, and a control filter to calculate corrections to the aircraft's inertial navigation system (INS). This is accomplished by correlating terrain profiles from a radar altimeter with candidate digital map terrain profiles in the surrounding area, and selecting the most similar digital map profile in obtaining horizontal position (refs. 1-3). Stored terrain data have also been used in trajectory and guidance generation. Applications range from simple straight-line terrain following between waypoints to sophisticated "ground-hugging" meandering flight (ref. 4).

Digital terrain elevation database-dependent avionics are operationally constrained due to navigation and terrain database inaccuracies. As lower altitudes are approached and more aggressive maneuvering attempted, the ability of the aircraft to reliably and accurately position itself relative to the ground becomes vital. Unrecorded features and map horizontal shifts have been observed in flight tests (ref. 5). Persistent above-ground-level (AGL) bias due to navigation and terrain map errors must be identified and accounted for, in addition to higher frequency terrain features unrepresented in the stored terrain database.

A low-level, maneuvering terrain following/terrain avoidance (TF/TA) guidance system for helicopters has been under development at NASA Ames Research Center (ref. 6). The guidance algorithm uses mission requirements, aircraft performance capabilities, navigation data, and digitized terrain elevation data to generate a lowaltitude, valley-seeking trajectory. This trajectory is generated in real-time and presented to the pilot on a helmet-mounted display. The system's flight envelope is principally limited in its ability to position itself within the terrain, and its inability to detect and avoid unmapped obstacles, such as trees and wires. After evaluation in several full-motion, piloted simulations, the system has reached sufficient maturity for flight evaluation. A joint NASA/Army program to flight test the system on the U.S. Army NUH-60 STAR (Systems Testbed for Avionics Research) helicopter is scheduled for spring 1992.

An appraisal of the digital terrain map prior to flight evaluation was conducted to establish a minimum clearance altitude during flight tests (ref. 7). This was accomplished by comparing predicted terrain elevation database values based on measured horizontal position with elevation obtained by taking the difference between measured navigational vertical position and radar altitude. Precision navigation (from a ground-station improved Global Positioning System (GPS) receiver) and radar altimeter returns were recorded as a test aircraft flew low-altitude missions over rugged and plain areas. The combined navigation and database errors in terrain elevation were found to be as great as $220 \mathrm{ft}$, establishing a minimum clearance altitude for flight test of $220 \mathrm{ft}$ AGL.

To improve AGL positioning of the NASA/Army terrain database dependent guidance system, a Kalman filter which augments this system through the integration of a radar altimeter is developed. Radar altimeter measurements are blended with inertial navigation system vertical position and stored digital terrain data to provide a more accurate estimate of altitude AGL than that based solely on airborne navigation and terrain elevation data. The estimate is also more robust and stable than that provided by a radar altimeter alone. The linear, sequential measurement processing Kalman filter presented is found to essentially remove the AGL positioning limitation of the aforementioned guidance system, leaving only the flight envelope constraint imposed by obstacle detection and avoidance. 
The paper begins with the problem formulation and system description. State models are then developed and the Kalman filter cast. Filter performance is appraised using helicopter flight data acquired over moderately rugged terrain, and concluding remarks are then given.

\section{Problem Formulation}

Figure 1 describes key variables and definitions involved in the low-altitude, digital terrain map referenced flight environment.

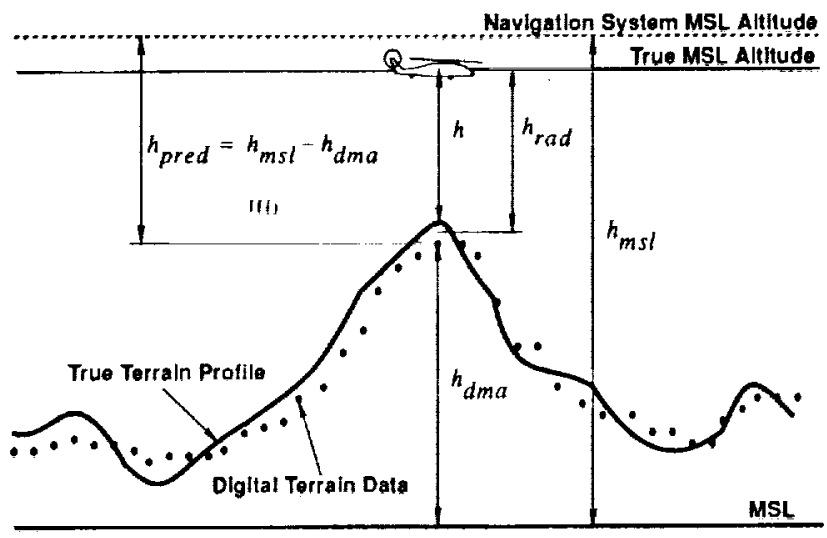

Figure 1. Problem description.

The aircraft is depicted on a nominal flight path with altitude above-ground-level denoted as $h$. Navigational mean-sea-level (MSL) altitude is denoted as $h_{m s}$, and sampled terrain elevation data as $h_{d m a}$. The difference in these two values is the "predicted" AGL altitude, the current method of determining height above ground. The radar altimeter measurement for AGL altitude is represented as $h_{\mathrm{rad}}$. This measurement, along with the predicted measurement of AGL altitude, is to be blended to yield an improved estimate of $h$.

A block diagram of the integrated system is shown in figure 2 .

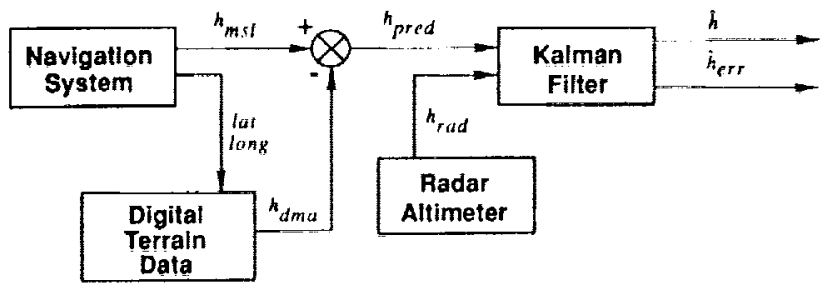

Figure 2. System block diagram.
There are two inputs to, and two outputs from, the Kalman filter. The first input, the predicted AGL altitude, $h_{\text {pred }}=h_{m s l}-h_{d m a}$, has multiple error sources. Regardless of the navigation system employed, $h_{m s}$ will contain some error. GPS would provide the most accurate and reliable positioning solution. The civilian Coarse Acqui sition code (C/A-code) of GPS gives positioning accuracy of $20-40 \mathrm{~m}(66-131 \mathrm{ft})$, degrading to $100 \mathrm{~m}(328 \mathrm{ft})$ when Selective Availability is activated. The military Precision

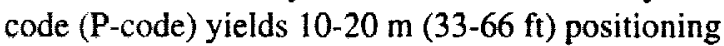
accuracy. Differential GPS yields 2-3 m (7-10 ft) positioning accuracy (ref. 8).

The accessed value for terrain elevation, $h_{d m a}$, is an imperfect approximation of the terrain, and is referenced using the imperfect latitude-longitude output from the navigation system. A Level 1 Defense Mapping Agency (DMA) Digital Terrain Elevation Data (DTED) database consists of a uniform matrix of MSL terrain elevation values. These database values have an "acquracy objective" of $30 \mathrm{~m}(98 \mathrm{ft}$ ) at $90 \%$ linear error for absolute

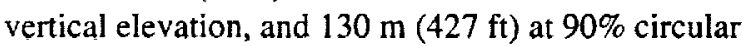
error for absolute horizontal position. Each $1 \mathrm{deg}$ by $1 \mathrm{deg}$ latitude-longitude cell carries its own accuracy specifications, however, which depend on the data collection method used for that area, and can be greater than the general database accuracy objectives. Because the terrain elevation stored in the DMA database is accessed through the latitude-longitude value of the navigation system, horizontal positioning errors will reference offset terrain data. The sum of this $h_{d m a}$ error, combined with those of the navigation system, can lead to large errors in the predicted absolute AGL altitude, although relative, lower frequency AGL altitude movement will be fairly accurate and reliable.

The second input to the Kalman filter of figure 2 is the radar altimeter, a direct measurement of the aboveground-level altitude. Typical radar altimeters are limited in operational altitude and degrade in accuracy with altitude. Most are fan-type, i.e., a conical beam is transmitted, and height above ground or nearest obstacle is returned. The measurement is thus relatively insensitive to aircraft roll and pitch attitude, and returns height above the nearest terrain feature. The spreading of the radar beam "footprint," however, can yield radar altimeter returns registered from nearby higher terrain, rather than that directly below the aircraft. Flight over a dense forest will yield height above the treetops (canopy height), while flight over bare (winter) trees will give height above the ground (refs. 9 and 10).

These two measurements are then blended in a Kalman filter to yield an improved estimated value for AGL altitude, $h_{t}$. As a by-product, the error between this 
AGL estimate and the predicted AGL value, i.e., $h_{e r r}=h_{p r e d}-h$, will also be produced.

\section{State Models}

Two states variables are defined:

$$
\begin{aligned}
& x_{1}=h \\
& x_{2}=h_{\text {err }}=h_{\text {pred }}-h
\end{aligned}
$$

The first state is the AGL altitude, and the second is the time-varying bias error between the first state and the predicted (navigation/terrain database) AGL altitude.

The first state is modeled as a random walk:

$$
\dot{x}_{1}=w_{1}
$$

where $w_{1}$ is white noise of zero mean and variance $\sigma_{1}^{2}$, i.e., $w_{1} \sim N\left[0, \sigma_{1}^{2}\right]$. The white noise standard deviation was set to $20 \mathrm{ft} / \mathrm{sec}$. Physically, this describes a system driven by white noise. This rather simplistic model was taken due to this state's strong dependence on flight profile. The AGL altitude signal would be quite different for an aircraft flying a low-level (constant MSL) mission over hilly terrain versus a contour (constant AGL) flight over the same area. Terrain characteristics (flat versus mountainous) would also generate different AGL altitude traces. More sophisticated, perhaps mission dependent AGL altitude state models could be considered in future work.

Two models for the second state $h_{e r r}$ will be considered. The first model is that of a first-order Gauss-Markov process described by:

$$
\dot{x}_{2}=-\beta x_{2}+\sqrt{2 \sigma_{2}^{2} \beta} w_{2}
$$

where $1 / \beta$ is the process time constant and $w_{2}$ is white noise of $w_{2} \sim N\left[0, \sigma_{2}^{2}\right]$. A Gauss-Markov process is a stationary Gaussian process (all probability density functions are normal) with an exponential autocorrelation of $E\left[x_{2}(t) x_{2}(t+\tau)\right]=\sigma_{2}^{2} e^{-\beta|\tau|}$. This presents a slowly varying model for the coupled navigational and terrain database errors. Spectral analysis of flight data established $1 / \beta$ at $10 \mathrm{sec}$ and $\sigma_{2}$ at $45 \mathrm{ft} / \mathrm{sec}$. Note that a $1 / \beta$ of $10 \mathrm{sec}$ corresponds to an along-track distance of $\sim 1 / 4$ mile for an aircraft flying at $90 \mathrm{kts}$.

An alternative model for the $h_{e r r}$ state is that of a random-ramp process described by:

$$
\begin{aligned}
& \dot{x}_{2}=x_{3}+w_{2} \\
& \dot{x}_{3}=w_{3}
\end{aligned}
$$

with white noise inputs of $w_{2} \sim N\left[0, \sigma_{2}^{2}\right]$ and $w_{3} \sim N\left[0, \sigma_{3}^{2}\right]$. The standard deviations for both $w_{2}$ and $w_{3}$ were set at $10 \mathrm{fu} / \mathrm{sec}$. Such a slope-intercept model naturally leads to an additional state variable. Physically, this model may more accurately represent the $h_{e r r}$ state over dense foliage contained in a valley. In this situation, the radar altimeter would typically return height above the treetops. Because trees commonly grow to a uniform altitude in a valley, while a DMA terrain elevation database stores height of the valley floor, a ramp model for the AGL altitude error seems reasonable. (Consider an aircraft flying at constant MSL altitude: its radar altimeter would measure a constant AGL altitude to the treetops, while the navigation/terrain database $h_{p r e d}$ would register the sloping valley floor.)

Finally, the two measurements are defined as:

$$
\begin{aligned}
z_{1} & =h_{m s l}-h_{d m a} \\
& =x_{1}+x_{2}+v_{1} \\
z_{2} & =h_{r a d} \\
& =x_{1}+v_{2}
\end{aligned}
$$

where the instrument errors $v_{1}$ and $v_{2}$ are Gaussian white noise of $v_{1} \sim\left[0, \sigma_{1}^{2}\right]$ and $v_{2} \sim\left[0, \sigma_{2}^{2}\right]$. The standard deviations were set at $10 \mathrm{ft} / \mathrm{sec}$ and $20 \mathrm{ft} / \mathrm{sec}$ for $v_{1}$ and $v_{2}$, respectively.

It should be noted that the state equations are attempting to model AGL altitude given two measurement sources with distinctly different characteristics. The first measurement, $h_{m s l}-h_{d m a}$, will give good relative height-aboveground information. This measurement is expected to be quite smooth and reliable, although it will carry a bias due to both the navigation vertical position solution and the stored DMA map. The radar altimeter complements the navigation/terrain database measurement in registering higher frequency absolute height-above-ground movements. This measurement, however, will be somewhat noisy and of higher variance than measurement one. The Kalman filter serves to blend these two measurements in producing a more stable, responsive, and accurate estimate of AGL altitude.

The explicit separation of the by-product $h_{e r r}$ in the state equations allows for flexibility in planned flight test implementation of the filter. Both filter outputs of AGL altitude and the difference in AGL altitude (between this estimate and that based on navigation/terrain database) are thus readily available.

These linear state models are now written in discrete-time, state-space form for each of the two models for the second 
state $h_{e r r}$. Using the Gauss-Markov model for $h_{e r r}$, one obtains

$$
\left[\begin{array}{l}
x_{1} \\
x_{2}
\end{array}\right]_{k+1}=\left[\begin{array}{cc}
1 & 0 \\
0 & e^{-\beta \Delta t}
\end{array}\right]\left[\begin{array}{l}
x_{1} \\
x_{2}
\end{array}\right]_{k}+\left[\begin{array}{l}
\omega_{1} \\
\omega_{2}
\end{array}\right]_{k}
$$

and for the measurements

$$
\left[\begin{array}{l}
z_{1} \\
z_{2}
\end{array}\right]_{k}=\left[\begin{array}{ll}
1 & 1 \\
1 & 0
\end{array}\right]\left[\begin{array}{l}
x_{1} \\
x_{2}
\end{array}\right]_{k}+\left[\begin{array}{l}
v_{1} \\
v_{2}
\end{array}\right]_{k}
$$

Similarly, the alternative random-ramp state model for $h_{\text {err }}$ yields the state equation:

$$
\left[\begin{array}{l}
x_{1} \\
x_{2} \\
x_{3}
\end{array}\right]_{k+1}=\left[\begin{array}{ccc}
1 & 0 & 0 \\
0 & 1 & \Delta t \\
0 & 0 & 1
\end{array}\right]\left[\begin{array}{l}
x_{1} \\
x_{2} \\
x_{3}
\end{array}\right]_{k}+\left[\begin{array}{l}
w_{1} \\
w_{2} \\
w_{3}
\end{array}\right]_{k}
$$

and measurement

$$
\left[\begin{array}{l}
z_{1} \\
z_{2}
\end{array}\right]_{k}=\left[\begin{array}{lll}
1 & 1 & 0 \\
1 & 0 & 0
\end{array}\right]\left[\begin{array}{l}
x_{1} \\
x_{2} \\
x_{3}
\end{array}\right]_{k}+\left[\begin{array}{l}
v_{1} \\
v_{2}
\end{array}\right]_{k}
$$

\section{Kalman Filter}

The discrete-time Kalman filter is a recursive optimal control filter most appropriate for estimating a noisy signal given noisy measurements. The Kalman optimal criterion is the minimization of mean-square error. The gains which satisfy this criterion are computed for each measurement sample. These gains take into account prior performance of measurements and states, in addition to a priori statistical knowledge of the random processes present. The filter is formulated using the notation of Brown (ref. 11). State and measurement equations are written as:

$$
\begin{gathered}
x_{k+1}=\Phi_{k} x_{k}+\omega_{k} \\
z_{k}=H_{k} x_{k}+v_{k}
\end{gathered}
$$

where $\Phi_{k}$ is the state transition matrix, $H_{k}$ is the observation matrix, and $w_{k}$ and $v_{k}$ are uncorrelated white noise.

The updating of the state equations for given measurements is accomplished by constantly computing the error covariance matrix

$$
P_{k}=E\left[e_{\bar{k}} e \frac{T}{k}\right]
$$

where $e_{\bar{k}}=x_{k}-\hat{x}_{\bar{k}}$ is the estimation error, and $\hat{x}_{\bar{k}}$ is the estimated value of $x$ based on all measurements up to, but not including, those at step time $t_{k}$.

Statistical properties of the white noise sequences $w_{k}$ and $v_{k}$ are defined by the covariance matrices

$$
\begin{gathered}
Q_{k}=E\left[w_{k} w_{k}^{T}\right] \\
R_{k}=E\left[v_{k} v_{k}^{T}\right]
\end{gathered}
$$

The state and measurement white noise disturbances are uncorrelated, i.e., $E\left[w_{k} v_{i}^{T}\right]=0$ for all $k$ and $i$.

After initial values $\hat{x}_{o}, P_{o}$ for the states and error covariance are established, the recursive Kalman filter equations are:

$$
\begin{aligned}
K_{k} & =P_{k} H_{k}^{T}\left[H_{k} P_{k} H_{k}+R_{k}\right]^{-1} \\
\hat{x}_{k} & =\hat{x}_{\bar{k}}+K_{k}\left[z_{k}-H_{k} \hat{x}_{\bar{k}}\right] \\
P_{k} & =\left[I-K_{k} H_{k}\right] P_{k}
\end{aligned}
$$

where $K_{k}$ is the Kalman gain matrix, $\hat{x}_{k}$ is the updated state estimate given measurements through $z_{k}$, and $P_{k}$ is the updated error covariance matrix. The initial state values, $x_{o}$, were set with respect to the first measurements received, equivalent to setting the elements of $P_{o}$ at infinity.

The state and error covariance matrices are projected ahead to the next time step as:

$$
\begin{aligned}
& \hat{x}_{\bar{k}+1}=\Phi_{k} \hat{x}_{k} \\
& P_{k+1}=\Phi_{k} P_{k} \Phi_{k}^{T}+Q_{k}
\end{aligned}
$$

In actual operation where time steps are asynchronous, these projections occur upon receipt of the measurements, to allow the actual time increment to be used.

The Kalman filter is implemented to process the measurements sequentially, an established procedure (refs. 12 and 13) which allows a measurement rejection test to be applied. The structure of the filter matrix equations (18)-(22) remains unchanged, but the measurement matrix $H_{k}$ becomes a row vector and the measurement covariance matrix $R_{k}$ becomes a scalar corresponding to the scalar measurement $z_{k}$ being processed.

The rejection test compares each measurement with that predicted from the measurement model

$$
\hat{z}_{k}=h_{k} \hat{x}_{\bar{k}}
$$


whereby a measurement deemed statistically unreasonable is thrown out and not used to update the state and error covariance matrices. The measurement residual

$$
\rho_{k}=z_{k}-\hat{z}_{k}
$$

is compared with the expected standard deviation of that measurement

$$
\alpha_{k}=\sqrt{h_{k} P_{k} h_{k}^{T}+r_{k}}
$$

in determining acceptance of a measurement.

In this work, a two standard deviation $\left(2 \alpha_{1}\right)$ criterion was established for $\rho_{1}$ (residual from navigation/terrain database predicted AGL altitude measurement) and a $4 \alpha_{2}$ criterion for $\rho_{2}$ (radar altimeter measurement residual). Thus, if $\rho_{1}$ exceeded $2 \alpha_{1}$, or if $\rho_{2}$ was greater than $4 \alpha_{2}$, that measurement was discarded. These thresholds were set based on the behavior of the instruments used in acquiring the flight test data considered in this report, and reflect a greater confidence in the radar altimeter measurement than the navigation/terrain database measurement. Such rejection limits would have to be adjusted for different measurement sources than those considered here, and possibly for flight conditions (e.g., poor GPS satellite navigation data due to satellite geometry or intermittent reception).

Finally, for numerical stability the symmetric error covariance matrix $P_{k}$ was forced to remain symmetric after every measurement update by averaging the offdiagonal elements. Divergence of a Kalman filter without such a constraint is well documented (refs. 11 and 12).

\section{Test Procedure}

The performance of the Kalman filter is assessed using low-altitude helicopter flight test data. Both models for the second state $h_{e r r}$ are considered, allowing a direct comparison of their relative performance to be made. Inputs required are aircraft position (MSL altitude, latitude, longitude) from a navigation system, radar altimeter return, and digital terrain elevation data.

The flight test data were acquired by the U.S. Army Avionics Research and Development Activity (AVRADA) in support of the terrain-referenced navigation research of Hollowell (ref. 3) during fall 1989. The same data were employed in this author's earlier work (ref. 7) on digital terrain elevation data, which describes the flight characteristics and instruments in greater detail than are given here. Low-altitude helicopter flights were conducted in a UH-1 (Huey) helicopter in south-central Pennsylvania, just south of Harrisburg. The moderately rough terrain varied from flat plain sections through mountainous regions containing regions of dense deciduous trees. The aircraft flew at a constant $\sim 90 \mathrm{kts}$.

Airborne data recorded at $1 \mathrm{~Hz}$ included 4-channel C/A code GPS navigation (Motorola Eagle Mini-Ranger), with specified $25 \mathrm{~m}(82 \mathrm{ft}$ ) positioning accuracy. Selected availability, the intentional degradation of the GPS signal, was not activated. A nearby ground station, at a known surveyed site with an identical GPS receiver, provided differential GPS (DGPS) bias corrections. These corrections were applied during postprocessing to the airborne GPS navigation solution for improved helicopter positioning. Such a procedure requires the ground and airborne receivers to be tracking the same constellation of satellites. The positional DGPS procedure employed operates with the positional solution of the ground receiver, rather than the more common DGPS technique that uses raw pseudo-ranges received from the satellites (ref. 8).

Radar altimeter data were recorded at $1 \mathrm{~Hz}$. The fan-type radio-frequency altimeter (Honeywell APN-209) returned aircraft height above ground or closest terrain obstacle to altitudes of $1500 \mathrm{ft}$, and through pitch and roll angles of 45 deg. Flight over dense foliage typically results in height above the foliage canopy top, while flight over bare trees gives height above the ground itself. Radar altimeter accuracy was specified to be $3 \mathrm{ft}+3 \%$ of actual altitude (refs. 9 and 10).

The terrain elevation database employed was Level 1 DMA DTED in the 1 deg by $1 \mathrm{deg}$ cell from $77 \mathrm{deg}$ to $78 \mathrm{deg}$ west longitude and from $40 \mathrm{deg}$ to $41 \mathrm{deg}$ north latitude. The database carried accuracy specifications of $260 \mathrm{~m}(853 \mathrm{ft})$ in absolute horizontal position and $50 \mathrm{~m}$ (164 ft) in absolute vertical elevation, both at $90 \%$ confidence level. The database prediction of terrain elevation is found by forming a triangular plane of the nearest three "posts" of DMA data. The interpolated value of this plane below the aircraft is taken as the database elevation prediction.

\section{Results and Discussion}

The Kalman filter estimate of AGL altitude, $\hat{h}_{t}$, is shown in figure 3 for a typical section of flight data. During this period, the helicopter was maintaining a constant MSL altitude of $\sim 1000 \mathrm{ft}$, over terrain ranging from $300 \mathrm{ft}$ to $700 \mathrm{ft}$ MSL. 
The two measurements are also shown in figure 3 . The abscissa represents a downrange distance of $\sim 5.5 \mathrm{n}$. mi., corresponding to an aircraft speed of $\sim 90 \mathrm{kts}$. Despite the coupled measurement model ( $H$ non-diagonal in eqs. (10) and (12)), the estimate $\hat{h}_{t}$ was essentially the same for the two $h_{e r r}$ models of equations (4)-(6). Hence, only one of the estimates for $\hat{h}_{t}$ is given in figure 3 .

The amount of error contained in an AGL altitude computed from on-board navigation and stored terrain elevation data (i.e., measurement $z_{1}$ ) is striking when compared with the AGL altitude sensed by the radar altimeter $\left(z_{2}\right)$. For instance, at time $25 \mathrm{sec}$ the predicted AGL altitude $\left(z_{1}\right)$ puts the aircraft's AGL altitude at $413 \mathrm{ft}$, whereas the radar altimeter puts it at $513 \mathrm{ft}$. Such a $110 \mathrm{ft}$ disparity in these two measurements clearly illustrates the need for improvement to an AGL altitude determined solely from airborne navigation and a stored terrain map.

Sections of flight data dropout are evident during the period shown in figure 3 . At time $12 \mathrm{sec}$ for $10 \mathrm{sec}$ and again at time $65 \mathrm{sec}$ for $7 \mathrm{sec}$ there were no navigational or radar altimeter data recorded. During these periods, the state estimate is dictated by the state transition matrix $\Phi_{k}$ of equation (13). State propagation is based on the last updates of the error covariance $P_{k}$ and associated Kalman gains, $K_{k}$. The constant $\hat{h}_{t}$ value during these periods is a consequence of the random walk model for $h_{t}$.

The effect of the rejection test implemented in the sequential measurement processing Kalman filter is evident in figure 3. Just prior to the data dropouts mentioned, erroneous navigation MSL data were generated by the GPS receiver, namely at times $12 \mathrm{sec}$ and $65 \mathrm{sec}$. At these locations the navigation vertical solution was deemed unreasonable and dismissed, i.e., the measurement residual of equation (25) exceeded the twice standard deviation criterion set for $z_{1}$. The filter did not update the state, error covariance, and gain matrices with these measurements. Possible explanations for these GPS "wild points" would include GPS signal multi-pathing, antenna blockage, or receiver queries for better satellite constellations. The radar altimeter measurement did fall within its established acceptance limits at these points, however, and was used for updating.

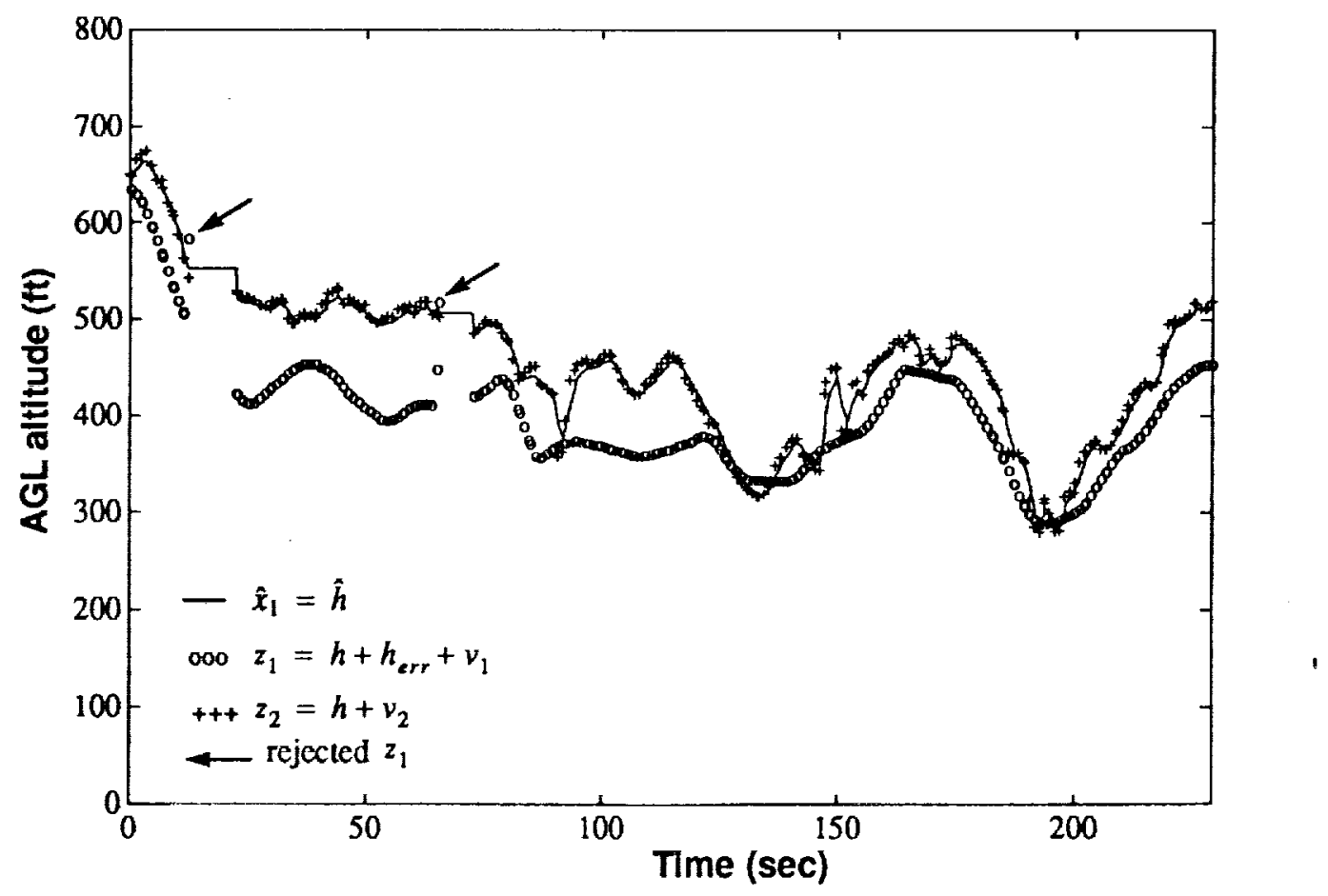

Figure 3. Filter AGL altitude output. 


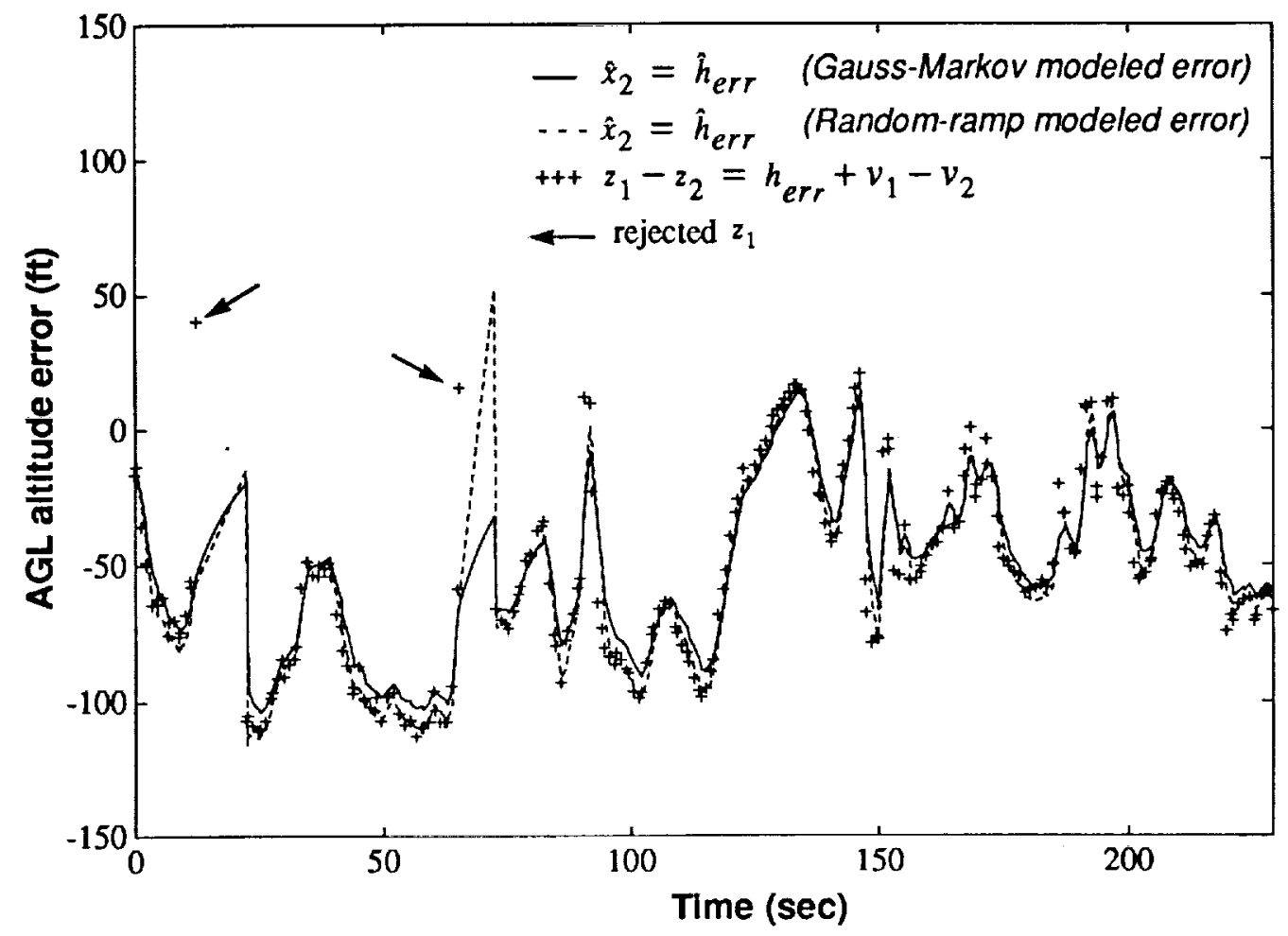

Figure 4. Filter AGL altitude error output.

The filtered estimate of $\hat{h}_{\text {err }}$ over the same section of flight test data is shown as figure 4.

The difference in the two measurements, $z_{1}-z_{2}$, is plotted with the filter estimate $\hat{h}_{e r r}$. The output for each of the two mathematical state models for $h_{\text {err }}$ is shown. Recall the data discussed at time $25 \mathrm{sec}$ in figure 3 , which indicated a predicted AGL altitude $z_{1}$ of $413 \mathrm{ft}$ and radar altimeter AGL $z_{2}$ of $523 \mathrm{ft}$. These data are reflected in figure 4 where $z_{1}-z_{2}$ is $-0 \mathrm{ft}$ for time $25 \mathrm{sec}$.

The performance of the Kalman filter in estimating $\hat{h}_{e r r}$ for each of the two state models is about the same, except for their marked difference during measurement dropouts. During these periods (time 12 to $22 \mathrm{sec}$, and time 65 to $73 \mathrm{sec}$ ) the fundamental difference between the GaussMarkov and random-ramp model is apparent. The GaussMarkov model of equation (9) yields an exponentially decaying $\hat{h}_{e r r}$ value in the absence of additional measurements. The random ramp model in equation (11), however, projects a ramp function. As such, the more stable Gauss-Markov model for the state $h_{e r r}$ is preferred.

Both state models for the AGL error $\left(h_{e r r}\right)$ rejected the same erroneous predicted AGL altitude measurements $\left(z_{1}\right)$ referred to earlier. Differences between estimated state $\hat{h}_{e r r}$ and the measurement of $h_{e r r}=z_{1}-z_{2}$ never exceeded $50 \mathrm{ft}$ during this and the additional 90 min of flight data analyzed. The filtered estimate of true AGL altitude $\hat{h}_{t}$ could be used directly for AGL positioning in an exclusively navigation/terrain map-based system, or the by-product state $\hat{h}_{e r r}$ could be added to the existing system's predicted AGL altitude.

The figures presented are representative of the kind of accuracy, responsiveness, stability, and robustness of the filter for the helicopter flight test data considered.

\section{Concluding Remarks}

1) A Kalman filter for the integration of a radar altimeter into a terrain database-dependent guidance system has been developed. The filter's performance was analyzed using low-altitude helicopter flight test data acquired over moderately rugged terrain. The two-state Kalman filter is stable, responsive, and accurate in estimating both the aircraft above-ground-level (AGL) altitude, and the by-product AGL altitude difference error, present in the existing terrain-based system.

2) The sequential measurement processing Kalman filter performs well in the presence of measurement anomalies and data dropouts. An input data rejection test has been implemented which successfully identifies and disregards erroneous data based on statistical criteria. 
3) The radar altimeter augmented near-terrain system allows for reduced minimum clearance altitude operation to approximately $50 \mathrm{ft}$, subject to obstacle avoidance limitations. Without the radar altimeter integration, terrain database and navigational errors restricted operation to $220 \mathrm{ft}$. Flight is now limited as a function of pathway obstacles, e.g., trees and wires, which would have to be identified by a forward-looking sensor.

The NASA/Army near-terrain guidance system, with the proposed radar altimeter integration, is scheduled for flight evaluation during spring 1992. A forward-looking sensor for obstacle detection and avoidance will eventually be incorporated as nap-of-the-earth operation is approached.

The author would like to thank Ray Clark et al. (U.S. Army AVRADA) for providing the flight test data and documentation.

\section{References}

1. Baird, C.; and Abramson, M.: A Comparison of Several Digital Map-Aided Navigation Techniques. Proceedings of the IEEE Position Location and Navigation Symposium, San Diego, CA, 1984.

2. Boozer, D.; and Fellerhoff, J.: Terrain-Aided Navigation Test Results in the AFTI/F-16 Aircraft. Navigation: Journal of the Institute of Navigation, vol. 35, no. 2, Summer 1988.

3. Hollowell, J.: A Terrain Referenced Navigation Algorithm for Helicopters. Proceedings of the IEEE Position Location and Navigation Symposium, Las Vegas, NV, March 1990.

4. Bair, G.; and Johnston, D.: Radar Systems Analysis Using DTED Data. Proceedings of the IEEE National Aerospace and Electronics Conference, Dayton, OH, 1987.
5. Nordmeyer, R.: Enhanced Terrain Masked Penetration Final Technical Report. AFWALTR-86-1079, Sept. 1986.

6. Swenson, H. N.; Zelenka, R. E.; Hardy, G.; and Dearing, M.: Simulation Evaluation of a LowAltitude Helicopter Flight Guidance System. Proceedings of the 10th IEEE/AIAA Digital Avionics Systems Conference, Los Angeles, CA, Oct. 14-17, 1991.

7. Zelenka, R. E.; and Swenson, H. N.: Appraisal of Digital Terrain Elevation Data for Low-Altitude Flight. Proceedings of the American Helicopter Society 48th Annual Forum, Washington, D.C., June 3-5, 1992.

8. McNally, B. D.; Paielli, R. A.; Bach, R. E., Jr.; and Warner, D. N.: Flight Evaluation of Differential GPS Aided Inertial Navigation Systems. AGARD Guidance and Control Panel Specialist Meeting on Integrated and Multi-Function Navigation, Ottawa, Canada, May 14-15, 1992.

9. Headquarters, Dept. of the Army: Technical Manual for Altimeter Set, Electronic. TM-11-5841-29213\&P, Nov. 1980.

10. Headquarters, Dept. of the Army: Military Specification Altimeter Set, Electronic AN/APN-209(V). MIL-A-49079A(CR), June 1986.

11. Brown, R. G.: Introduction to Random Signal Analysis and Kalman Filtering. John Wiley \& Sons, Inc., 1983.

12. Gelb, A.: Applied Optimal Estimation. The M.I.T. Press, 1984.

13. Kaminski, P.; Bryson, A.; and Schmidt, S.: Discrete Square Root Filtering: A Survey of Current Techniques. IEEE Transactions on Automatic Control, vol. AC-16, no. 6, Dec. 1971. 


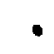



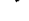

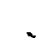


Public reporting burden for this collection of information is estimated to average 1 hour per response, Including the time for reviewing instructions, searching existing data sources gathering and maintaining the data needed, and completing and reviewing the collection of intormation. Send comments regarding this burden estimale or any other aspect of this Davis Highway, Suite 1204, Arlington, VA 22202-4302, and to the Office of Management and Budget, Paperwork Reduction Project (0704-0188), Washington, DC 20503.

\begin{tabular}{l|l|l} 
1. AGENCY USE ONLY (Leave blank) & $\begin{array}{c}\text { 2. REPORT DATE } \\
\text { August } 1992\end{array}$ & $\begin{array}{c}\text { 3. REPORT TYPE AND DATES COV } \\
\text { Technical Memorandum }\end{array}$
\end{tabular}

4. TITLE AND SUBTITLE

5. FUNDING NUMBERS

Integration of Radar Altimeter, Precision Navigation, and Digital Terrain

Dảta for Low-Altitude Flight

6. AUTHOR(S)

$505-64-13$

Richard E. Zelenka

7. PERForming ORganization NAME(S) AND ADdRESS(ES)

8. PERFORMING ORGANIZATION REPORT NUMBER

Ames Research Center

Moffett Field, CA 94035-1000

A-92158

9. SPONSORING/MONITORING AGENCY NAME(S) AND ADDRESS(ES)

10. SPONSORING/MONITORING AGENCY REPORT NUMBER

National Aeronautics and Space Administration

Washington, DC 20546-0001

NASA TM-103958

11. SUPPLEMENTARY NOTES

Point of Contact: Richard E. Zelenka, Ames Research Center, MS 210-9, Moffett Field, CA 94035-1000

(415) 604-5433

12a. DISTRIBUTION/AVAILABILITY STATEMENT

12b. DISTRIBUTION CODE

Unclassified - Unlimited

Subject Category 04

13. ABSTRACT (Maximum 200 words)

Avionic systems that depend on digitized terrain elevation data for guidance generation or navigational reference require accurate absolute and relative distance measurements to the terrain, especially as they approach lower altitudes. This is particularly exacting in low-altitude helicopter missions, where aggressive terrain hugging maneuvering create minimal horizontal and vertical clearances and demand precise terrain positioning. Sole reliance on airborne precision navigation and stored terrain elevation data for aboveground-level (AGL) positioning severely limits the operational altitude of such systems. A Kalman filter is presented which blends radar altimeter returns, precision navigation, and stored terrain elevation data for AGL positioning. The filter is evaluated using low-altitude helicopter flight test data acquired over moderately rugged terrain. The proposed Kalman filter is found to remove large disparities in predicted AGL altitude (i.e., from airborne navigation and terrain elevation data) in the presence of measurement anomalies and dropouts. Previous work suggested a minimum clearance altitude of $220 \mathrm{ft}$ AGL for a near-terrain guidance system; integration of a radar altimeter allows for operation of that system below $50 \mathrm{ft}$, subject to obstacle-avoidance limitations.

14. SUBJECT TERMS

Terrain referenced guidance, Kalman filter, Radar altimeter, Low-altitude flight, Digital terrain elevation data, Defense Mapping Agency (DMA) 15. NUMBER OF PAGES

\begin{tabular}{|l|c|c|c|}
\hline 17. SECURITY CLASSIFICATION \\
$\begin{array}{l}\text { OF REPORT } \\
\text { Unclassified }\end{array}$ & $\begin{array}{c}\text { 18. SECURITY CLASSIFICATION } \\
\text { OF THIS PAGE } \\
\text { Unclassified }\end{array}$ & $\begin{array}{l}\text { 19. SECURITY CLASSIFICATION } \\
\text { OF ABSTRACT }\end{array}$ & 20. LIMITATION OF ABSTRACT \\
\hline
\end{tabular}

